\title{
An autoregressive model for global vertebrate richness rankings: long-distance dispersers may have stronger spatial structures
}

Youhua Chen ${ }^{1,2}$

\begin{abstract}
Background: Spatial autocorrelations are one of the most prevalent natural phenomena in ecological data. It is generally assumed that short-distance dispersers are spatially limited and thus have stronger spatial autocorrelation patterns than do long-distance dispersers. To test this hypothesis, I quantified and compared spatial autocorrelation patterns of global richness rankings of amphibians, mammals, and birds using an autoregressive model. A species richness ranking was used as a proxy of species richness, which was obtained through a digital image processing method from published world maps of species richness.
\end{abstract}

Results: The results showed that the spatial structure could explain the highest variance involved in global richness rankings of mammals (intermediate-distance dispersers), followed by birds (long-distance dispersers). In contrast, amphibians, representing short-distance dispersers, had the lowest degree of spatial autocorrelation patterns. Thus, the present results do not support the abovementioned hypothesis.

Conclusions: In conclusion, a complex relationship exists between an animal's dispersal ability and its spatial autocorrelation pattern. The dispersal abilities of species can be negatively correlated with spatial autocorrelation patterns.

Keywords: Global richness patterns; Spatial autocorrelation; Autoregression models; Variance partitioning

\section{Background}

Spatial autocorrelations are a common phenomenon in ecological and evolutionary studies (Legendre 1993; Legendre and Legendre 1998). Many studies attempted to quantify and eliminate spatial autocorrelation signals to better evaluate the influence of environmental variability in shaping species community structures (Borcard et al. 1992; Iop et al. 2012), distributions (Dormann 2007; Dormann et al. 2007; Sullivan et al. 2012), and diversities (Tognelli and Kelt 2004; Diniz-Filho et al. 2009).

Generally, there are two forms of spatial autocorrelation: autocorrelations of environmental gradients and species' distributions. Herein, autocorrelations of species' distributions were specifically measured as the number of species found in each grid cell as used in previous studies

\section{Correspondence: haydi@126.com}

'Department of Zoology, University of British Columbia, Vancouver V6T 1Z4, Canada

${ }^{2}$ Current address: Department of Renewable Resources, University of Alberta, Edmonton T6G 2H1, Canada

\section{Springer}

(Tognelli and Kelt 2004; Vieira et al. 2008), or aggregated occupancy information of each single species. Spatial autocorrelations of species richness are derived from spatial autocorrelations of environmental variables and/or dispersal limitations of each single species. Because all examined vertebrate groups are constrained to the global terrestrial scale, it is assumed that the influence of spatial autocorrelations of environmental variables is basically equivalent over various vertebrate groups (because the distributions of all species are bound to the Earth's surface). As a consequence, it is supposed that resultant species richness patterns are principally driven by differences in dispersal abilities among taxonomic groups. Of course, this strong assumption cannot eliminate the fact that important environmental predictors can vary across different species, but the central goal of the present study was to reveal the effect of dispersal on species richness patterns.

Community ecology and macroecology argue that dispersal is strongly correlated with spatial autocorrelations (Bahn et al. 2008; Shurin et al. 2009; Flinn et al. 2010; 
Caruso et al. 2012; Curini-Galletti et al. 2012). One common associated assumption is that species exhibiting longdistance dispersal should be less influenced by space, since they can disperse across different habitats and choose the most suitable ranges for survival (Shurin et al. 2009; Flinn et al. 2010). Thus, it is predicted that the environment should determine their distribution and community structure. In contrast, short-distance dispersers are not able to occupy all suitable areas around the world and are typically constrained to narrow ranges (being spatially clumped). Thus, because of a limited dispersal ability, they should be less influenced by the environment compared to longdistance dispersers. So, short-distance dispersers are commonly presumed to be spatially limited and have stronger spatial autocorrelation patterns (Bahn et al. 2008; Shurin et al. 2009; Flinn et al. 2010). This hypothesis has been confirmed in plant (Flinn et al. 2010) and freshwater (Shurin et al. 2009) communities.

Because ectothermic vertebrates are thought to have poorer dispersal abilities compared to endothermic vertebrates (Cook et al. 2000; Chen et al. 2012; Tingley and Dubey 2012), it is hypothesized in the present study that global richness patterns of birds (long-distance dispersers) should have less of a spatial autocorrelation structure, followed by mammals (intermediate-distance disperses). In comparison, the global richness of amphibians (shortdistance dispersers) should possess the strongest spatial autocorrelation signal. This hypothesis was established based on a mean field approximation. That is, complex spatial autocorrelation patterns of global richness patterns of species assemblages are determined by the simple spatial autocorrelation pattern derived from the distribution of each single species in that assemblage.

In the present report, I developed a simple and novel digital image processing algorithm to obtain rough global richness patterns. The algorithm can extract color information (in terms of red, green, and blue channels) from previously published world maps of species richness. Through $k$-means clustering, this color information extracted from pixels of the map can be classified into different classes to represent richness rankings of species, which can serve as a proxy for species richness. Then, using a simple autoregressive model, I revealed global patterns of spatial autocorrelations in richness rankings of amphibians, birds, and mammals and tested the above hypothesis linking dispersal abilities and spatial structure patterns of species assemblages.

\section{Methods}

Global richness rankings of amphibians, birds, and mammals Worldwide richness patterns of birds, mammals, and amphibians were extracted and standardized using published digital maps, which were collected from a previous report (Grenyer et al. 2006). Extraction of species richness in each grid cell was difficult because the digitized map had limited resolution and the colors representing different species richness values were difficult to match. Thus, I considered an alternative option and used a richness ranking as a proxy to study the relationship of space and species diversity. The $k$-means clustering method was employed to obtain 15 classes of species richness based on the extracted digitized color information (in terms of red, green, and blue channels) from the map pixels. Because not all classes of the pixel colors could represent major richness gradients of terrestrial species and some may represent species richness in small areas, marginal areas, or oceanic islands, each color class was checked and compared by eye to obtain major richness rankings at a continental scale based on the published literature (Grenyer et al. 2006). Some grid cells for which it was difficult to determine their richness rankings or that were located on islands and at edges of territory were not considered. As such, I obtained 12 rankings for birds, 9 for amphibians, and 8 for mammals. The clustering algorithm was implemented using $\mathrm{R}$ software ( $\mathrm{R}$ Development Core Team 2011). Figure 1 demonstrates and explains the procedures of color extraction and clustering in detail to obtain species richness rankings from a hypothetical species richness map.

When the spatial autoregression analysis was carried out on the richness ranking data of the present study, a spatial weighting square matrix, $W$, was incorporated. During the analysis, computational difficulty was encountered: in my original datasets, there respectively were 44,319, 38,955, and 42,623 sampling points for birds, amphibians, and mammals (data available upon request). With these original data, the $W$ matrix would have become a super-large sparse matrix, which would have been hard to generate and calculate. In view of this, I considered a resampling process to obtain new rankings of the three taxonomic groups with a coarser spatial grid resolution of $6^{\circ} \times 6^{\circ}$. Thus, the resultant datasets for birds, amphibians, and mammals respectively contained 529,510 , and 520 sample grid cells. The richness ranking of each new grid cell was recalculated by averaging those from the original points, which had to fall within the new grid cell.

\section{Measure of the spatial autocorrelation}

The spatial autocorrelation was analyzed using Moran's $I$ index as follows:

$$
I=\frac{n}{S} \frac{\sum_{i=1}^{n} \sum_{j=1}^{n} w_{i j}\left(x_{i}-\bar{x}\right)\left(x_{j}-\bar{x}\right)}{\sum_{i=1}^{n}\left(x_{i}-\bar{x}\right)^{2}}
$$

where $n$ is the number of quadrats (or grid cells) in the study, $x_{i}$ and $x_{j}$ represent values of species richness in 


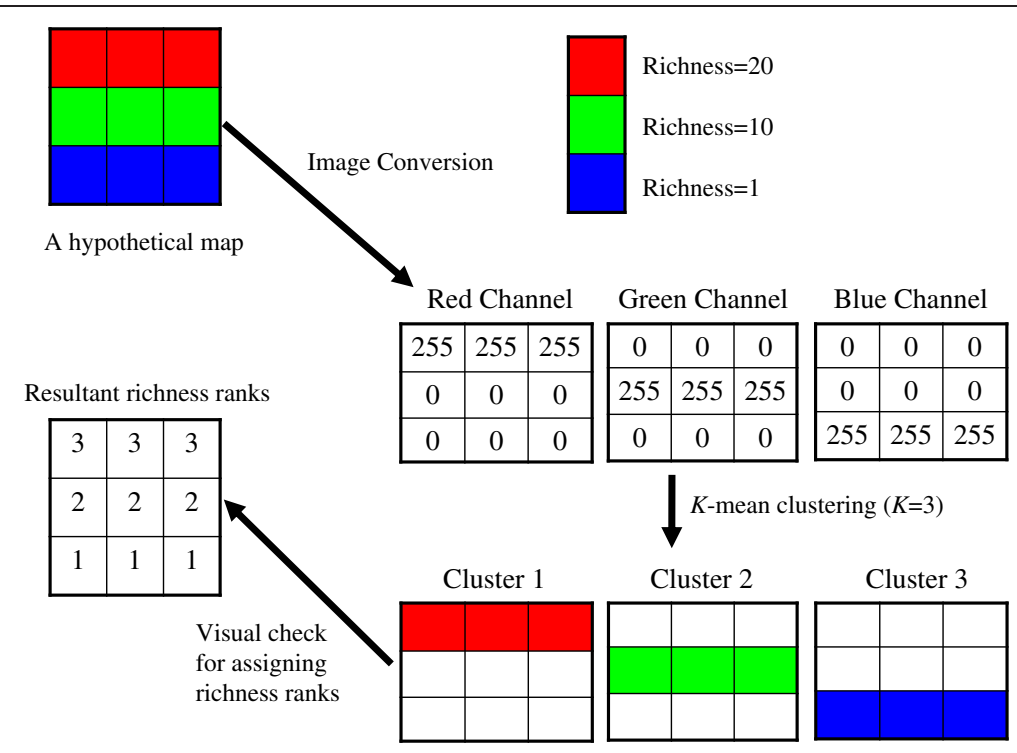

Figure 1 Illustration of the color extraction and classification procedures. The procedures in the present study were used for generating species richness ranks of grid cells from a hypothetical richness map. The core of this procedure was to convert the color from each of the grid cells in the map into red, green, and blue (RGB) channels with numeric values ranging $0 \sim 255$. The $k$-means clustering method was then applied to the resultant RGB color channel matrices to reconstruct clusters of groups for the RGB numeric data. Finally, a naked-eye check of the numeric data grouping was carried out to match the richness classes in the original and hypothetical richness maps. After the visual check, a richness rank was assigned to each of the grid cells to obtain the resultant richness rank map, which was used for the subsequent autoregressive analyses.

quadrats $i$ and $j, \bar{x}$ is the mean of all $x$, and $w_{i j}$ is an element in the weighting matrix, $W$. It can be defined as follows:

$$
w_{i j}=\left\{\begin{array}{cc}
1 & i, j \text { is within a given distance class } \\
0 & \text { otherwise }
\end{array} .\right.
$$

This weighting matrix, $W$, is called a binary matrix based on the definition of its elements $w_{i j}$ using Equation 2 .

Finally, $S$ represents a normalizing factor as follows:

$$
S=\sum_{i=1}^{n} \sum_{j=1}^{n} w_{i j}
$$

\section{Autoregressive spatial modeling}

There are a number of autoregressive models (Tognelli and Kelt 2004; Dormann et al. 2007), and the simplest one I used was adopted from previous works (Diniz-Filho et al. 1998; Dormann 2007; Vieira et al. 2008; Diniz-Filho et al. 2012):

$$
y=\rho W y+\varepsilon
$$

where $W$ is the row-standardized weighting matrix, $\rho$ is the autoregressive parameter (which I set to $0 \leq \rho \leq 1$ ), and $\varepsilon$ is an error vector. This model can be fitted by maximum-likelihood procedures. The squared correlation between the observed richness ranking, $y$, and the estimated $\hat{y}=\rho W y$ gives the pseudo- $R^{2}$ of the model, showing the proportion of explained variance attributed to the spatial autoregressive process.

There are numerous possible weighting matrices, $W$; a simple one is the matrix identical to that defined in Moran's $I$ formula as in Equation 2. Another option for the weighting matrix, $W$, was derived from a previous study (Vieira et al. 2008), each element of which is written as

$$
w_{i j}=1 / D_{i j}^{a}
$$

where $D_{i j}$ is the geographic distance between the centroids of grid cells $i$ and $j$. Various values of $a$, ranging $1 \sim 1.5$ with steps of 0.1 , were tested in this study (other values of $>1.5$ are possible, but I found that the likelihood fitting for $\rho$ would exceed the upper bound of 1). I called this $W$ matrix constructed from geographic distances the distance matrix.

\section{Model evaluation}

Autoregressive models with different weighting matrices were also tested and compared using different methods (Vieira et al. 2008). $R^{2}$ values of the model indicate the ability of each model to explain the spatial structure of the species richness ranking, whereas an autocorrelation analysis based on Moran's $I$ index in the residual term, $\varepsilon$, indicate the effectiveness of the model for removing the spatial autocorrelation structure. The Akaike information criterion (AIC) (Akaike 1974) was also used to 
choose the best model. For each model, the modified AIC $\left(\mathrm{AIC}_{\mathrm{C}}\right)$ corrected for small samples is computed as (Haining 2003):

$$
\mathrm{AIC}_{\mathrm{C}}=2 n \log \left(\sigma^{2}\right)+2 K \frac{n}{n-K-1},
$$

where $n$ is the number of cells, $K$ is the number of parameters in the model, and $\sigma^{2}$ is the variance of the residuals of each regression model, so the first term above was positive in an effort to minimize the variance. The variance of the residuals was used here as a proxy for the likelihood of the model given the data. The term $\frac{n}{n-K-1}$ is a small sample correction and tends toward 1 as $n$ increases. I compared $\mathrm{AIC}_{\mathrm{C}}$ values of each model using the difference between the AIC of each model and the minimum $\mathrm{AIC}_{\mathrm{C}}$ found. A value of $>10$ indicates that the model has a poor fit relative to the best model, whereas a value of $<3$ indicates that the model is equivalent to the best model (Vieira et al. 2008).

\section{Results}

Patterns of spatial autocorrelations in global richness rankings of amphibians, mammals, and birds

The spatial autocorrelation signal was greatest for mammals and lowest for amphibians (Table 1), based on the fact that the pseudo- $R^{2}$ was highest while the $\mathrm{AIC}_{\mathrm{C}}$ was lowest for mammals.

Increasing the power factor, $a$, when computing the $W$ matrix using Equation 5, increased the spatial autocorrelation signal in the richness rankings, as evidenced by an increasing pseudo- $R^{2}$, a decreasing $\mathrm{AIC}_{\mathrm{C}}$ value, and an increasing Moran's $I$ index. This pattern was universal across all three vertebrate taxonomic groups (Table 1).
Analogously, increasing the sampling neighboring distance classes when computing the $W$ binary matrix using Equation 2 also increased the spatial autocorrelation signal in species richness rankings. The patterns were basically similar to cases of increasing the power factor, $a$, except for the situation with Moran's $I$ index: when the sampling neighboring classes exceeded six, it basically remained unchanged even when neighboring classes increased.

When comparing the distance and binary $W$ matrix models, it was found that the distance $W$ matrices explained a greater amount of the variation. Thus, the distance weighting matrix performed better than the binary neighboring weighting matrix in modeling the spatial autocorrelation signal in species richness patterns. This pattern was not compared or identified in a previous work (Vieira et al. 2008), in which only different network models were compared to construct a $W$ matrix.

Long-distance dispersers can have a strong spatial structure When comparing amphibians and birds, birds are definitely better dispersers than amphibians. However, contradictory to the abovementioned hypothesis that shortdistance dispersers should have stronger spatial autocorrelation patterns, birds were found to have a stronger spatial autocorrelation pattern compared to amphibians in the present results. Furthermore, although the dispersal ability of mammals is weaker than that of birds, and mammals have the strongest spatial effect, the explained variance attributed to the spatial autocorrelation (indicated by fitting determination $R^{2}$ ) for birds was slightly smaller than that for mammals (Table 1). The explained variance became almost identical when only cases for the binary $W$ matrix were considered.

Table 1 Comparison of autoregressive models with different $W$ for assessing global patterns of spatial autocorrelations

\begin{tabular}{|c|c|c|c|c|c|c|c|c|c|c|c|c|}
\hline \multirow[t]{2}{*}{$w$} & \multicolumn{4}{|c|}{ Mammals } & \multicolumn{4}{|c|}{ Amphibians } & \multicolumn{4}{|c|}{ Birds } \\
\hline & $\rho$ & $\mathrm{AIC}_{\mathrm{C}}$ & $R^{2}$ & Moran's I & $\rho$ & $\mathrm{AIC}_{\mathrm{C}}$ & $R^{2}$ & Moran's I & $\rho$ & $\mathrm{AlC}_{\mathrm{C}}$ & $R^{2}$ & Moran's I \\
\hline \multicolumn{13}{|c|}{ Distance matrix } \\
\hline$a=1$ & 0.118 & $7,443.883$ & 0.914 & 0.184 & 0.126 & $8,034.76$ & 0.784 & 0.206 & 0.117 & $8,693.137$ & 0.862 & 0.153 \\
\hline$a=1.1$ & 0.17 & $7,414.297$ & 0.916 & 0.213 & 0.182 & $7,972.835$ & 0.797 & 0.238 & 0.169 & $8,664.672$ & 0.866 & 0.18 \\
\hline$a=1.2$ & 0.242 & $7,382.141$ & 0.919 & 0.243 & 0.261 & $7,906.554$ & 0.81 & 0.27 & 0.241 & $8,631.682$ & 0.87 & 0.208 \\
\hline$a=1.3$ & 0.341 & 7,347.771 & 0.921 & 0.274 & 0.369 & $7,836.91$ & 0.822 & 0.304 & 0.341 & $8,594.431$ & 0.874 & 0.237 \\
\hline$a=1.4$ & 0.476 & $7,311.783$ & 0.924 & 0.306 & 0.516 & $7,765.194$ & 0.834 & 0.338 & 0.478 & $8,553.433$ & 0.879 & 0.268 \\
\hline$a=1.5$ & 0.658 & $7,274.965$ & 0.927 & 0.338 & 0.714 & $7,692.925$ & 0.846 & 0.372 & 0.662 & $8,509.428$ & 0.884 & 0.299 \\
\hline \multicolumn{13}{|l|}{ Binary matrix } \\
\hline Class $=2$ & 0.003 & $9,385.66$ & 0.441 & -0.031 & 0.003 & $9,273.898$ & 0.274 & -0.021 & 0.004 & $10,170.65$ & 0.442 & 0.016 \\
\hline Class $=4$ & 0.003 & $9,385.66$ & 0.441 & -0.031 & 0.003 & $9,273.898$ & 0.274 & -0.021 & 0.004 & $10,170.65$ & 0.442 & 0.016 \\
\hline Class $=6$ & 0.003 & $9,305.439$ & 0.483 & 0.011 & 0.003 & $9,211.798$ & 0.316 & 0.03 & 0.003 & $10,094.25$ & 0.481 & 0.045 \\
\hline Class $=8$ & 0.003 & $9,305.439$ & 0.483 & 0.011 & 0.003 & $9,211.798$ & 0.316 & 0.03 & 0.003 & $10,094.25$ & 0.481 & 0.045 \\
\hline Class $=10$ & 0.003 & $9,305.439$ & 0.483 & 0.011 & 0.003 & $9,211.798$ & 0.316 & 0.03 & 0.003 & $10,094.25$ & 0.481 & 0.045 \\
\hline
\end{tabular}

$\rho$, autoregressive parameter; $\mathrm{AlC}_{C}$ modified Akaike information criterion; $R^{2}$, the proportion of explained variation; $a$, the parameter in Equation 5. 


\section{Discussion}

Herein, I report that when spatial scales are (uplifted/ increased/expanded?), the resultant explained variance (indicated by $R^{2}$ ) attributed to the spatial autocorrelation should accordingly increase. Any scaling-up processes for generating either larger sampling grids when creating the richness ranking vector, $y$, or more neighbors when creating the weighting matrix $W$ (especially in binary cases) will result in increasing the spatial autocorrelation signal in species richness rankings (Table 1). This phenomenon was observed at various spatial scales, including local (He and Duncan 2000; Legendre et al. 2009), regional (mammal and bird distributions in the Cerrado region of Central Brazil (Vieira et al. 2008)), and global (the present study) ones.

My study contrasted with the common understanding that long-distance dispersers should be less influenced by space (i.e., environmentally structured), while dispersallimited species should be more influenced by space (Bahn et al. 2008; Shurin et al. 2009; Flinn et al. 2010). Instead, long-distance-dispersing species could have a better spatial structure than short-distance-dispersing species, as evidenced by comparing the explained variance for birds and amphibians. The dispersal ability of species may be inaccurate for predicting spatial autocorrelation patterns, which was found across different taxa, including animals, plants, and parasites (Harrison et al. 1992; Poulin 2003). Moreover, a previous field study showed that local dispersal did not contribute to the spatial autocorrelation of mistletoe richness per tree (Overton 1996). At a broad spatial scale, long-range dispersal was found to be important in structuring species diversity patterns. For example, long-distance dispersal was vital to the interplay of climatic conditions in determining the spread of the gypsy moth (Tobin and Blackburn 2007).

A likely explanation for the present observation that long-distance dispersers are more spatially structured may be linked to surrounding environmental conditions. If a species' distribution is primarily determined by environmental variables such as climate, which are spatially structured, then its distribution may have a stronger spatial autocorrelation than a dispersal-limited species that is restricted to small habitat patches with little spatial structure (at the coarse resolution of this study). Such an explanation can be comparatively tested if environmental information is included.

However, long-distance dispersers do not necessarily exhibit stronger spatial autocorrelation patterns when evaluating their distributions. As evidenced by the present results (Table 1), the highest spatial structure was observed for global mammals, although their dispersal abilities are weaker than those of birds (Chen et al. 2012). This observation might be attributed to other life history traits of vertebrates. For example, slow growth rates can allow species to have strong spatial autocorrelation patterns (Bahn et al. 2008). Mammals are slowly growing species compared to birds and amphibians, suggesting that they could have strong spatial autocorrelation structures.

However, even though the richness patterns of mammals had the highest spatial structure, it is worth noting that the fitting powers for birds and mammals were basically identical with the binary $W$ matrices (Table 1 ). Thus, the assumption that longer-distance dispersers possess higher spatial autocorrelation structures is still valid for the current observations. This statement is further supported by a previous work (Vieira et al. 2008): birds were found to have a higher fitting determination $R^{2}$ than mammals in the Cerrado region of central Brazil. As such, it can be further predicted that the environment might have less influence on long-dispersing species. Actually, it was shown that there was a complex relationship between dispersal and spatial autocorrelation (Steinitz et al. 2006; Bahn 2008), and the dispersal ability of a species cannot solely determine its spatial autocorrelation because dispersal is a mechanism driven by various factors (Buchi and Vuilleumier 2012) which may or may not lead to a spatial autocorrelation. Incorporating other factors, including but not limited to those of the environment, disturbances, habitat heterogeneity, functional traits, and phylogeny, might also be important for maintaining spatial autocorrelations (Overton 1996; Kallimanis et al. 2006; Zhang et al. 2011; Buchi and Vuilleumier 2012).

It is possible that using richness rankings as a proxy of richness would lose some information, but major patterns in the data are still maintained, as evident from the consistency in the present study and previous reports ( $\mathrm{He}$ and Duncan 2000; Vieira et al. 2008; Legendre et al. 2009) of identifying the positive effect of long-distance dispersers on promoting spatial autocorrelation patterns. Moreover, when only digitized maps are available, classifying different richness classes using the $k$-means clustering algorithm is highly accurate and fast. Indeed, it is almost impossible to extract true richness numbers of species in each grid cell of published world maps because of such problems as color matching, resolution, and reconstruction.

Using richness classes allows one to reveal patterns of spatial autocorrelation in species richness gradients across the world. It was observed in the present study that distributions of global amphibians and mammals were more clustered than that of global birds, indicating that spatial autocorrelation signals of dispersal-limited species are more significant than those of long-distance dispersers.

There are certain limitations to the present study. First, it contained no environmental information of species richness data. Although it was previously assumed that spatial autocorrelation patterns of environmental variables were similar for the three vertebrate groups (because their distributions are bounded by the same 
environmental conditions worldwide), a comparative test of the influences of environmental filtering on different taxonomic groups can offer a holistic view of spatial autocorrelation signals in global species richness patterns. Thus, global environmental variables should be incorporated in future studies. Second, because richness rankings of species were extracted from published digital maps, some color information may have been lost during the extraction procedure, and the resultant reconstruction of species richness rankings might not truly reflect the global species richness of vertebrates. Therefore, the presently observed spatial autocorrelations of species richness rankings can be further reevaluated using highresolution spatial ranges of species.

\section{Conclusions}

There is a complex relationship between an animal's dispersal ability and its spatial autocorrelation pattern. The dispersal abilities of species can be negatively correlated with spatial autocorrelation patterns. Species richness ranking can be used as a surrogate of species richness, which can be extracted through a simple digital image processing method from published geographic maps of species richness.

\section{Competing interests}

The author declares that he has no competing interests.

\section{Acknowledgements}

I am very grateful to the editors and two anonymous referees for their constructive and critical comments that greatly improved the quality of this work. I also appreciate Dr. Rana Sarfraz for his help with polishing of the language. This work was partially supported by the University of British Columbia.

Received: 20 December 2012 Accepted: 4 September 2013

Published: 12 December 2013

\section{References}

Akaike $H$ (1974) Information theory as an extension of the maximum likelihood principle. In: Petrov BN, Caski F (ed) Proceeding of the second international symposium on information theory. Akademiai Kiado, Budapest, pp 276-281

Bahn V (2008) Failure to find the relationship between dispersal and spatial autocorrelation in species abundance. J Negat Results Ecol Evol 5:1-13

Bahn V, Krohn W, O'Connor R (2008) Dispersal leads to spatial autocorrelation in species distributions: a simulation model. Ecol Model 213:285-292

Borcard D, Legendre P, Drapeau P (1992) Partialling out the spatial component of ecological variation. Ecology 73:1045

Buchi L, Vuilleumier S (2012) Dispersal strategies, few dominating or many coexisting: the effect of environmental spatial structure and multiple sources of mortality. PLoS One 7:e34733

Caruso T, Taormina M, Migliorini M (2012) Relative role of deterministic and stochastic determinants of soil animal community: a spatially explicit analysis of oribatid mites. J Anim Ecol 81:214-221

Chen S, Zhang J, Wang X, Sun J, Xue Y, Zhang P, Zhou H, Qu L (2012) Extremely low genetic diversity indicating the endangered status of Ranodon sibiricus (Amphibia: Caudata) and implications for phylogeography. PLoS One 7:e33378

Cook R, Flather C, Wilson K (2000) Faunal characteristics of the southern Rocky Mountains of New Mexico: implications for biodiversity analysis and assessment: US Department of Agriculture (USDA) Forest Service General Technical Report RMRS-GTR-58.60. USDA, Fort Collins, Colorado
Curini-Galletti M, Artois T, Delogu V, De Smet W, Fontaneto D, Jondelius U (2012) Patterns of diversity in soft-bodied meiofauna: dispersal ability and body size matter. PLoS One 7:e33801

Diniz-Filho A, Nabout C, M de Campos Telles M, Sares T, Rangel TFLVB (2009) A review of techniques for spatial modeling in geographical, conservation and landscape genetics. Genet Mol Biol 32:203-211

Diniz-Filho J, Bii L, Rangel TF, Morales-Castilla I, Olalla-Tárraga M, Rodriguez M, Hawkins B (2012) On the selection of phylogenetic eigenvectors for ecological analyses. Ecography 35:239-249

Diniz-Filho J, Sant'Ana C, Bini L (1998) An eigenvector method for estimating phylogenetic inertia. Evolution 52:1247-1262

Dormann C (2007) Effects of incorporating spatial autocorrelation into the analysis of species distribution data. Glob Ecol Biogeogr 16:129-138

Dormann C, McPherson J, Araujo M, Bivand R, Bolliger J, Carl G, Davies R, Hirzel A, Jetz W, Kissling WD, Kühn I, Ohlemüller R, Peres-Neto PR, Reineking B, Schröder B, Schurr FM, Wilson R (2007) Methods to account for spatial autocorrelation in the analysis of species distributional data: a review. Ecography 30:609-628

Flinn K, Gouhier T, Lechowicz J, Waterway M (2010) The role of dispersal in shaping plant community composition of wetlands within an old-growth forest. J Ecol 98:1292-1299

Grenyer R, Orme CDL, Jackson SF, Thomas GH, Davies RG, Davies TJ, Jones KE, Olson VA, Ridgely RS, Rasmussen PC, Ding TS, Bennett PM, Blackburn TM, Gaston KJ, Gittleman JL, Owens IP (2006) Global distribution and conservation of rare and threatened vertebrates. Nature 444:93-96

Haining R (2003) Spatial data analysis: theory and practice. Cambridge University Press, Cambridge

Harrison S, Ross S, Lawton J (1992) Beta-diversity on geographic gradients in Britain. J Anim Ecol 61:151-158

He F, Duncan R (2000) Density-dependent effects on tree survival in an old-growth Douglas-fir forest. J Ecol 86:676-688

lop S, Caldart VM, Dos Santos TG, Cechin SZ (2012) What is the role of heterogeneity and spatial autocorrelation of ponds in the organization of frog communities in southern Brazil? Zool Stud 51:1094-1104

Kallimanis AS, Kunin WE, Halley JM, Sgardelis SP (2006) Patchy disturbance favours longer dispersal distance. Evol Ecol Res 8:529-541

Legendre P (1993) Spatial autocorrelation: trouble or new paradigm? Ecology 74:1659-1673

Legendre P, Legendre L (1998) Numerical ecology. Elsevier, Amsterdam, The Netherlands

Legendre P, Mi X, Ren H, Ma K, Yu M, Sun I-F, He F (2009) Partitioning beta diversity in a subtropical broad-leaved forest of China. Ecology 90:663-674

Overton J (1996) Spatial autocorrelation and dispersal in mistletoes: field and simulation results. Vegetatio 125:83-98

Poulin R (2003) The decay of similarity with geographical distance in parasite communities of vertebrate hosts. J Biogeogr 30:1609-1615

R Development Core Team (2011) R: a language and environment for statistical computing. , Vienna, Austria. Available at http://www.R-project.org. Accessed 09 October 2013. ISBN 3-900051-07-0

Shurin J, Cottenie K, Hillebrand H (2009) Spatial autocorrelation and dispersal limitation in freshwater organisms. Oecologia 159:151-159

Steinitz O, Heller J, Tsoar A, Rotem D, Kadmon R (2006) Environment, dispersal and patterns of species similarity. J Biogeogr 33:1044-1054

Sullivan M, Davies R, Reino L, Franco A (2012) Using dispersal information to model the species-environment relationship of spreading non-native species. Meth Ecol Evol 3:870-879

Tingley R, Dubey S (2012) Disparity in the timing of vertebrate diversification events between the northern and southern hemispheres. BMC Evol Biol 12:244

Tobin P, Blackburn L (2007) Long-distance dispersal of the gypsy moth (Lepidoptera: Lymantriidae) facilitated its initial invasion of Wisconsin. Environ Entomol 37:87-93

Tognelli M, Kelt D (2004) Analysis of determinants of mammalian species richness in South America using spatial autoregressive models. Ecography 27:427-436

Vieira C, Blmires D, Diniz-Fiho J, Bini L, Rangel TFLVB (2008) Autoregressive modelling of species richness in the Brazilian Cerrado. Braz J Biol 68:233-240

Zhang S, Slik J, Zhang JL, Cao K (2011) Spatial patterns of wood traits in China are controlled by phylogeny and the environment. Glob Ecol Biogeogr 20:241-250

doi:10.1186/1810-522X-52-57

Cite this article as: Chen: An autoregressive model for global vertebrate richness rankings: long-distance dispersers may have stronger spatial structures. Zoological Studies 2013 52:57. 